\title{
Fairness analysis of throughput and delay in WLAN environments with channel diversities
}

\author{
Chiapin Wang ${ }^{1 *}$, Hao-Kai Lo ${ }^{1}$ and Shih-Hau Fang ${ }^{2}$
}

\begin{abstract}
The article investigates fairness in terms of throughput and packet delays among users with diverse channel conditions due to the mobility and fading effects in IEEE 802.11 WLAN (wireless local area networks) environments. From our analytical results, it is shown that 802.11 CSMA/CA can present fairness among hosts with identical link qualities regardless of equal or different data rates applied. Our analytical results further demonstrate that the presence of diverse channel conditions can pose significant unfairness on both throughput and packet delays even with a link adaptation mechanism since the MCSs (modulation and coding schemes) available are limited. The simulation results validate the accuracy of our analytical model.
\end{abstract}

\section{Introduction}

Recently, IEEE 802.11 based wireless local area networks (WLAN) become increasingly prevailing with their ubiquitous nature and low cost infrastructure. The IEEE 802.11 standards [1] on medium access control (MAC) specify two fundamental mechanisms for channel arbitration, namely, distributed coordination function (DCF) and point coordination function (PCF). DCF is a random access mechanism based on the protocols of carrier sense multiple access with collision avoidance (CSMA/ $\mathrm{CA})$. PCF is a centralized scheduling protocol which uses a point coordinator at the access point (AP). Most of current 802.11 WLAN products employ DCF on account of its distributed nature for the simplicity of implementation [2]. To such networks, fairness is of particular concern since the overall system performance essentially depends on the allocation of transmission slots among users. It is considered over a short or long period of time separately for pertinently reflecting the performance of the specific applications or protocols. For example, the behavior of short-term fairness can make a significant impact on TCP transfers or delaysensitive multimedia applications [3]. In general, shortterm fairness means around an order of $10 \mathrm{~ms}$ scales while long-term fairness may involve a transmission of thousand packets [4].

\footnotetext{
* Correspondence: chiapin@ntnu.edu.tw

1Department of Applied Electronic Technology, National Taiwan Normal

University, Taipei, Taiwan

Full list of author information is available at the end of the article
}

Throughput and packet delays are two key measures reflecting the fairness performance of IEEE 802.11 WLANs. Bianchi [5] first proposed a Markov model for IEEE 802.11 DCF to evaluate saturation throughput. Ziouva et al. [6] improved Bianchi's model by considering that the backoff counter is frozen when the channel is sensed busy. Based on the above two works, Xiao [7] developed an analytical model for enhanced distributed coordination function (EDCF) of IEEE 802.11e WLAN [8]. However, most of these works analyzed throughput performance of DCF protocols in homogeneous PHY situations, i.e. equal data rates and identical channel conditions, which are inconsistent with practical WLAN environments. Wireless channels actually are time-varying due to fading, noise, interference, mobility etc., and therefore the varying channel conditions can affect the used data rates. Consequently, these previous analytical approaches considering only homogeneous PHY situations may be insufficient to tackle realistic WLAN environments.

The fairness performance of IEEE 802.11 WLAN is essentially affected by both the MAC-layer protocol and PHY channel diversity (also called multiuser diversity) [9], i.e., varied channel conditions among stations and unequal data rates determined by the applied link adaptation scheme. The authors in previous work [9-11] used experiments and simulations to study throughput performance of 802.11 DCF with channel diversities. However, they did not offer a complete theoretical analysis which can be the most important base for understanding the user-diversity impact on the system

\section{SpringerOpen ${ }^{\odot}$}

(C) 2011 Wang et al; licensee Springer. This is an Open Access article distributed under the terms of the Creative Commons Attribution License (http://creativecommons.org/licenses/by/2.0), which permits unrestricted use, distribution, and reproduction in any medium, provided the original work is properly cited. 
Table 1 The adopted IEEE $\mathbf{8 0 2 . 1 1 b}$ parameter.

\begin{tabular}{ll}
\hline Parameter & Value \\
\hline Slot-time & $20 \mu \mathrm{s}$ \\
SIFS & $10 \mu \mathrm{s}$ \\
DIFS & $50 \mu \mathrm{s}$ \\
Payload & 1023 bytes \\
PHY header & 24 bytes \\
MAC header & 28 bytes \\
ACK frame & 38 bytes \\
$C_{\text {min }}$ & 32 \\
$C_{\text {max }}$ & 1024 \\
Retry limit & 5 \\
\hline
\end{tabular}

performance of DCF. We are thus motivated to propose in this article an analytical model to analyze the fairness performance of DCF protocols with the cross-layer effects of PHY channel diversities. Our analytical approach is developed by extending a two-dimensional Markov chain model of DCF proposed by Bianchi [5] for considering time-varying channel conditions. However, our analytical model takes into account more realistic factors, including the finite retransmission limit, the probability that the backoff counter is frozen when the channel is sensed busy, error-prone channels, and also multiple data rates. Thus, by comparison with previous work $[5,7,12]$, our approach can more efficiently reflect the behavior of the present 802.11 protocols performing in realistic environments.

The contribution of this work is that we offer a theoretical model to thoroughly analyze the cross-layer impact of PHY channel diversities on the fairness performance of 802.11 DCF in terms of both throughput and packet delays. Through our analyses, it is shown that 802.11
CSMA/CA can present fairness only on condition that the link qualities of all the hosts are equal in a statistical average sense. It is also observed that diverse channel conditions can pose significant unfairness of both throughput and packet delays even with a link adaptation mechanism since MCSs (modulation and coding schemes) available are limited. We validate our analytical model via simulations and the results demonstrate its accuracy, showing the impracticality of providing performance analysis for DCF with only the consideration of homogeneous PHY conditions. The remainder of this article is organized as follows. In 'Related work' section, we discuss the related work. 'An analytical model of 802.11 DCF in error-prone channels' section, presents our analytical model of 802.11 DCF. In 'Validations' section, we validate the accuracy of this model via simulations. 'The numerical results and discussion' section shows analytical results which demonstrate the unfairness of 802.11 DCF due to diverse channel conditions. 'Conclusion' section draws our conclusions.

\section{Related work}

The fairness of IEEE 802.11 DCF has been largely studied with theoretical analyses, simulations, or experiments in previous work [2-4,10,13-35]. The fairness problems generally are discussed, respectively, with user-datagram-protocol (UDP) flows and transmissioncontrol-protocol (TCP) flows due to different behaviors of the two transport layer protocols. The considered transmission scenarios are further classified as two categories: transmissions among uplink flows or transmissions among uplink and downlink flows. A taxonomy table of the fairness problems in wireless networks is shown in Figure 1. With respect to UDP transmission

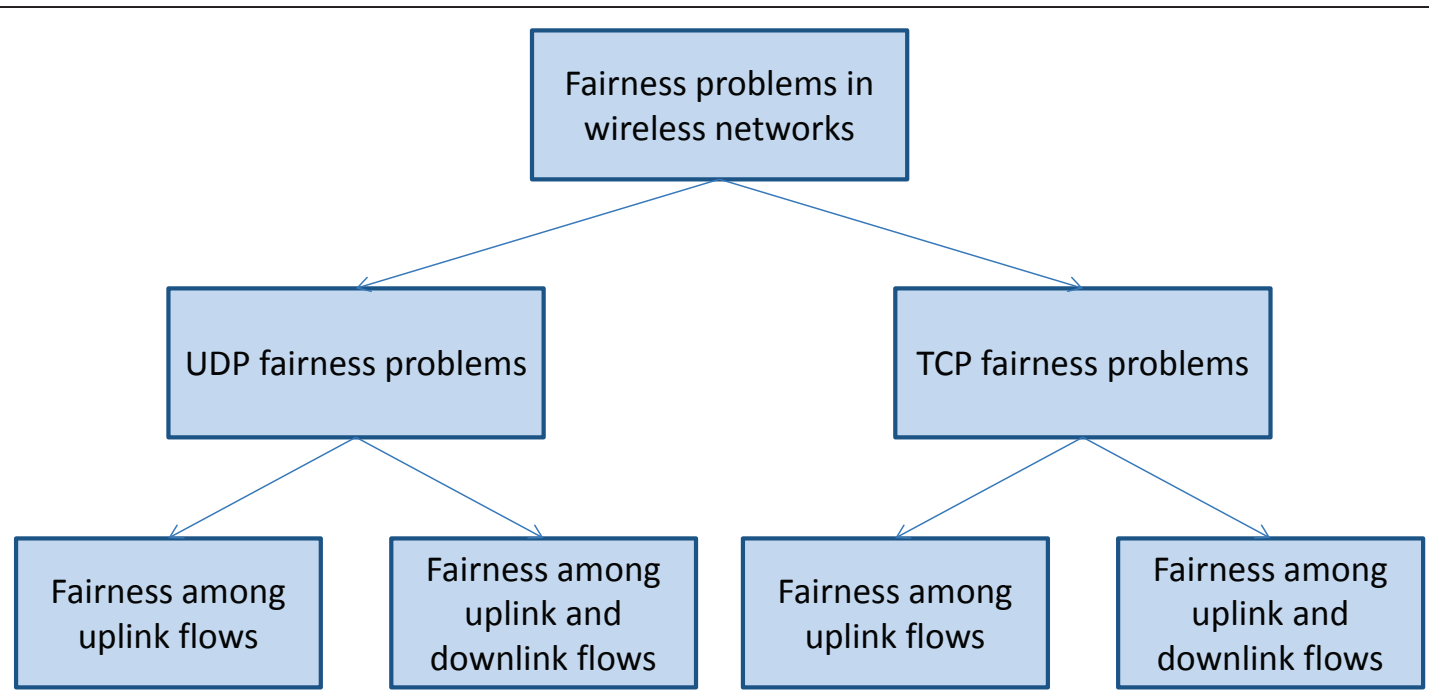

Figure 1 The classification of fairness problems in wireless networks. 
scenarios, the authors in [13] propose an approach that reduces AP's channel sensing time from DCF interframe space (DIFS) to PCF inter-frame space (PIFS) in order to meet the required utilization ratio for downlink traffic flows. This approach grants AP the highest priority to transmit its data frames immediately, but may cause the entire channel slots occupied by AP before the required utilization ratio is matched. The work [14] develops a new distributed contention control (DCC) algorithm that combines transient and stationary characteristics of slot utilization to better estimate congestion level of the medium. The work [15] presents a dynamic contention window control scheme based on the number of downlink flows to achieve per-flow fairness. Nevertheless, it does not consider some dynamics in WLAN environments such as channel conditions and traffic loads that can greatly impact the performance of fairness. The authors in [17] use an analytical approach to find optimal contention window sizes based on the observed idle slot intervals to achieve utility fairness between AP and wireless stations. However, the approaches proposed in [17] may need substantial modifications in the MAC layer protocols.

In general, the traffic load of downlink flows may be much heavier than that of uplink flows. The work in $[2,27,28]$ investigate weighted fairness in case that the downlink and uplink traffic loads are asymmetric. The authors in [2] present the bidirectional DCF (BDCF) which provides a preferential treatment to downlink traffic by piggybacking AP's data packets after acknowledge (ACK) frames. This approach can provide a ratio of downlink throughput to uplink throughput up to 1 . The work [27] develops adaptive schemes to achieve weighted fairness between uplink/downlink traffic flows by dynamically adjusting the backoff counters of AP and stations. The authors in [28] apply differentiated minimum contention windows $(\mathrm{CW})$ for AP and wireless stations to tune their channel utilization ratio.

The TCP unfairness problems in wireless networks have been researched in [29-33]. The work [29] provides a detail analysis of per-flow and per-station fairness for TCP flows. In [30] the authors propose a differentiated approach which involves multidimensional parameters including minimum CWs, arbitration inter-frame space (AIFS), and transmission opportunity (TXOP), to solve the TCP fairness problem between uplink and downlink traffic flows in 802.11e WLANs. The authors in [31] develop a cross-layer feedback approach to achieve per-station fairness by estimating each station's access time and queue length. The work [32] solves the TCP fairness problem by using a dual queue scheme in which one queue is specified for data packets of downlink TCP flows and the other is for ACK packets.
Most of the previous work present the observation that DCF is fair over long time scales but can not provide short-term fairness. Koksal et al. [3] argued that short-term unfairness is due to a phenomenon posed by the backoff protocol in CSMA/CA: a host capturing the channel will likely keep it after a contention period, which is similar to the well-known 'capture effect' shown in Ethernet [36]. However, Berger-Sabbatel et al. $[4,22]$ provided a contrary perception that DCF indeed presents pretty fine short-term fairness and consequently provides long-term fairness while short-term fairness implies long-term fairness, but not vice versa [3]. They argued that the confusion of fairness problem in the previous work [3] is as a result of using the CSMA/CA protocol specific to Wavelan system [37] instead of that characterized in 802.11 standards. Indeed, there is an important difference between the two access methods: the Wavelan CSMA/CA protocol executes exponential backoff when the channel is sensed busy, whereas 802.11 protocol does that only when a collision is experienced. Although the analysis of Berger-Sabbatel et al. $[4,22]$ is rather consistent with the behavior of the present 802.11 protocols, however, the conclusion is valid only under the assumption of homogeneous transmission qualities among the participating hosts, which may be unrealistic while hosts can experience unequal channel conditions due to mobility, fading, interference factors, and so on. Since an 802.11 exponential backoff performed is actually due to not only a transmission collision but also a packet corruption with bad signal qualities, the backoff behavior of hosts will be varied with their own link qualities, thereby leading to an unequal sharing of transmission channels.

\section{An analytical model of 802.11 DCF in error- prone channels}

In this section, we analyze IEEE 802.11 DCF protocols under UDP transmission scenarios by extending a twodimensional Markov chain model first proposed by Bianchi [5]. Our analytical model can be used to evaluate the statistical performance of DCF in realistic WLAN environments since it takes more factors into account including the finite retransmission limit, the probability that the backoff counter is frozen when the channel is sensed busy, error-prone channels, and multiple data rates. Furthermore, we provide performance analyses for both throughput and packet delay.

\subsection{Overview of IEEE 802.11 DCF}

First we briefly introduce 802.11 DCF based on CSMA/ CA. DCF consists of two access schemes, namely, basic scheme and four-way handshaking scheme. In the basic scheme, a host with a packet ready for transmission senses the medium first. While the medium is sensed 
idle for a period equal to a DCF inter-frame space (DIFS), the packet will be transmitted immediately. If the packet is then received successfully, the receiver host will send an acknowledgement (ACK) packet to the sender host after a short inter-frame space (SIFS). Otherwise, the sender host would choose an interval randomly from the backoff window before retransmitting the packet. The backoff counter is decremented in terms of slot time when the channel keeps idle. The counter is frozen when the channel is sensed busy and reactivated when the channel is sensed idle again for more than a DIFS. When the counter reaches 0 , the packet is retransmitted. If the packet retransmission is failed, the sender will increase its backoff window exponentially and perform another retransmission until the retry times come to a certain limit. In four-way handshaking scheme, the sender host transmits a request-tosend (RTS) packet first. If the receiver host hears RTS, it replies with a clear-to-send (CTS) packet. After receiving the CTS, the sender transmits the data packet. When successfully receiving the packet, the receiver replies with an ACK packet.

\subsection{The analytical model}

In this model, we consider $K$ IEEE 802.11 hosts in nonperfect channels. Assume that these hosts are within the transmission range of each other with each one always having a packet to send (i.e., operating in saturation conditions). To host $i(i=0$ to $K-1)$, let $p_{i, c}$ denote the probability of a packet collided with other hosts. That is:

$$
p_{i, c}=1-\prod_{h=0, h \neq i}^{K-1}\left(1-\tau_{h}\right),
$$

where $\tau_{h}$ is the probability for host $h$ transmitting a packet in a given slotted time. To host $i$, let $p_{i, e}$ denote the probability of a packet corrupted due to error-prone channels. $p_{i}$ e basically depends on SNR (signal-to-noise ratio), the used MCS, and the transmitting frame size [38]. Consider uncoded modulations like what are adopted from $802.11 \mathrm{~b}$ standards and assume that the BER (bit error rate) of host $i, p_{i, b}$ is unchanged inside each packet. Thus, $p_{i, e}$ can be expressed as:

$$
p_{i, e}=1-\left(1-p_{i, b}\right)^{\mathrm{FS}_{i} * 8},
$$

where $\mathrm{FS}_{i}$ is the frame size in bytes. To host $i$, the probability of a transmission failed, $p_{i, f}$, which consists of the probability of a packet collided and a collisionfree packet corrupted can be expressed as:

$$
p_{i, f}=p_{i, c}+\left(1-p_{i, c}\right) \cdot p_{i, e} .
$$

In 802.11 , a host needs to wait for a random backoff time before the next transmission to avoid a collision with other hosts. The random backoff timer is uniformly chosen in the interval $(0, \mathrm{CW}-1)$, where $\mathrm{CW}$ is the contention window size. After each retransmission due to a collision or a corruption, the CW will be doubled until the number of retries comes to a certain limit, $L_{\text {retry }}$ Let $\mathrm{CW}_{\text {min }}$ denote the initial $\mathrm{CW}$, and $\mathrm{CW}_{j}$ denote the $\mathrm{CW}$ in the $j$ th backoff stage. Once the $C W$ reaches a maximum value $C_{\text {max }}$, it will remain at the value until it is reset. Therefore, the relationships among $\mathrm{CW}_{j}, \mathrm{CW}_{\text {min }}$, $\mathrm{CW}_{\text {max }}$, and $L_{\text {retry }}$ are shown as follows:

$$
\begin{aligned}
& \mathrm{CW}_{j}= \begin{cases}2^{j} \mathrm{CW}_{\min } & \text { for } j=0,1, \ldots, m-1, \text { if } L_{\text {retry }}>m \\
2^{m} \mathrm{CW}_{\min }=\mathrm{CW}_{\max } & \text { for } j=m, \ldots ., L_{\text {retry }}, \text { if } L_{\text {retry }}>m \\
2^{j} \mathrm{CW}_{\min } & \text { for } j=0,1, \ldots, L_{\text {retry }}, \text { if } L_{\text {retry }} \leq m\end{cases} \\
& \text { where } m=\log _{2}\left(\mathrm{CW}_{\max } / \mathrm{CW}_{\min }\right)
\end{aligned}
$$

For host $i$, let $s(i, t)$ and $c(i, t)$ be the stochastic process representing the backoff stage and backoff time counter at time $t$, respectively. The two-dimensional process $\{s(i, t), c(i, t)\}$ can be modeled with the discretetime Markov chain shown in Figure 2. For the simplicity of illustration, we adopt the notation,

$$
P_{i}\left\{j_{1}, l_{1} \mid j_{0}, l_{0}\right\}=\operatorname{Pr}\left\{s(i, t+1)=j_{1}, c(i, t+1)=l_{1} \mid s(i, t)=j_{0}, c(i, t)=l_{0}\right\} .
$$

Thus, from the two-dimensional Markov chain we can obtain the following equations:

$$
\begin{cases}P_{i}\{j, l \mid j, l+1\}=1-p_{i, c}, & l \in\left(0, \mathrm{CW}_{j}-2\right), j \in\left(0, L_{\text {retry }}\right) \\ P_{i}\{0, l \mid j, 0\}=\left(1-p_{i, f}\right) / \mathrm{CW}_{\text {min }}, & l \in\left(0, \mathrm{CW}_{\text {min }}-1\right), j \in\left(0, L_{\text {retry }}-1\right) \\ P_{i}\{j, l \mid j-1,0\}=p_{i, f} / C W_{j}, & l \in\left(0, \mathrm{CW}_{j}-1\right), j \in\left(1, L_{\text {retry }}\right) \\ P_{i}\left\{0, l \mid L_{\text {retry }}, 0\right\}=1 / \mathrm{CW}_{\text {min }}, & l \in\left(0, \mathrm{CW}_{\text {min }}-1\right)\end{cases}
$$

The first equation in (5) represents the fact that the backoff counter is decremented when the channel is sensed idle with the probability of $\left(1-p_{i, c}\right)$ or frozen otherwise. The second equation accounts for the situation that a successful packet transmission with the probability of $\left(1-p_{i, f}\right)$ will return to backoff stage 0 and the counter is uniformly chosen in the interval $\left(0, \mathrm{CW}_{\mathrm{min}^{-}}\right.$ 1 ). The third equation considers the case of unsuccessful packet transmission that a retransmission due to collision or corruption will enter into the next backoff stage. Finally, the forth equation accounts for the fact that if the number of retries reaches the maximum value $L_{\text {retry, }}$, the backoff stage will be reset to 0 no matter the consequent transmission is successful or failed.

$$
\text { Let } b_{i, j, l}=\lim _{t \rightarrow \infty} \operatorname{Pr}\{s(i, t)=j, c(i, t)=l\}, \quad j \in\left(0, L_{\text {retry }}\right), \quad l \in\left(0, C W_{j}-1\right)
$$
be the stationary state probabilities of the Markov chain shown in Figure 2. From the chain regularity and by means of a simple computation, the following equations can be derived:

$$
\begin{aligned}
& b_{i, j, 0}=p_{i, f} \cdot b_{i, j-1,0} \rightarrow b_{i, j, 0}=p_{i, f}^{j} \cdot b_{i, 0,0}, \quad 0<j \leq L_{\text {retry }} \\
& b_{i, j, l}=\frac{\mathrm{CW}_{j}-l}{\mathrm{CW}_{j}} \cdot \frac{1}{1-p_{i, c}} \cdot b_{i, j, 0}, 0 \leq j \leq L_{\text {retry }}, 1 \leq l \leq \mathrm{CW}_{j}-1 .
\end{aligned}
$$




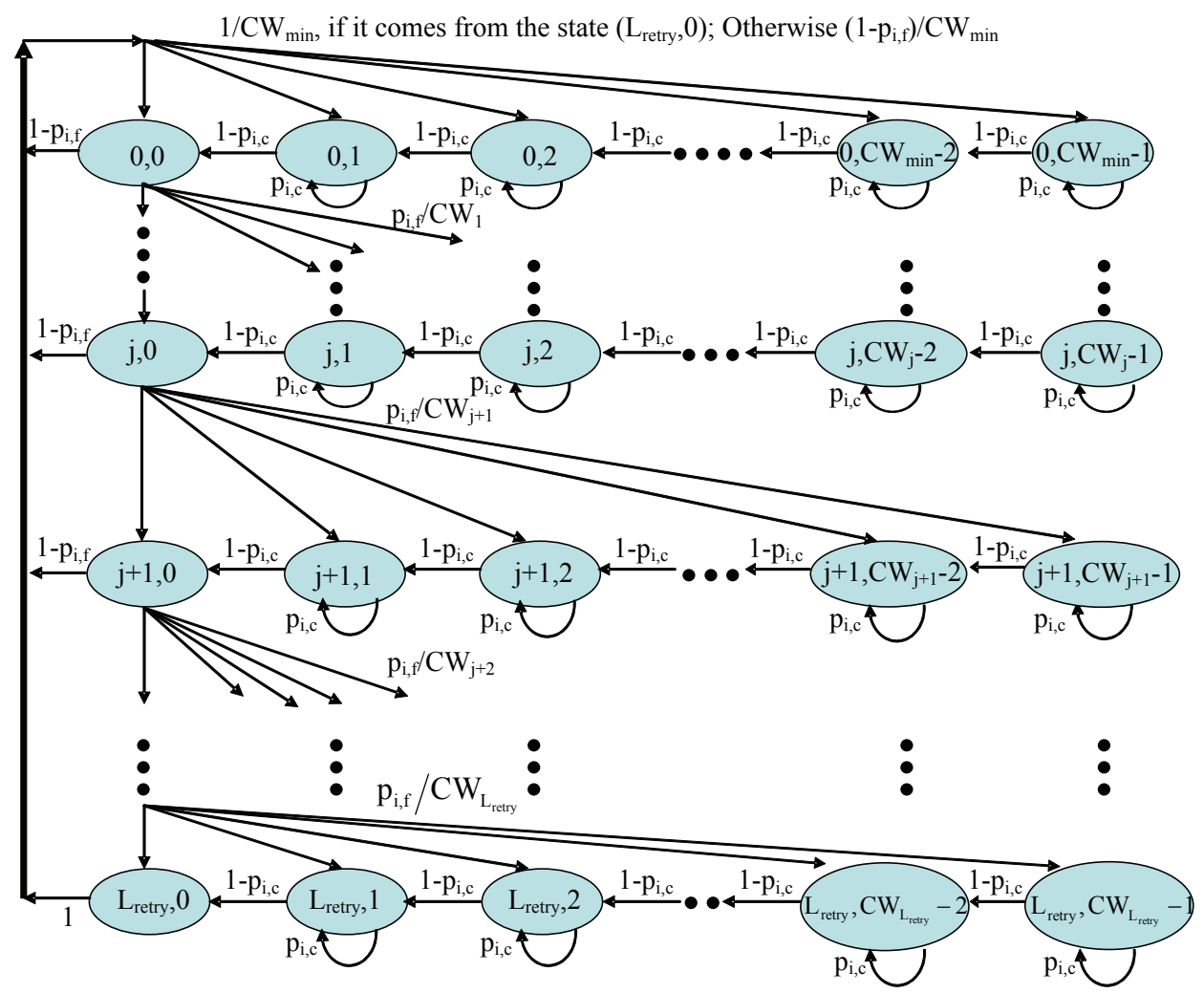

Figure 2 The state transition diagram of host $i$.

Equations 6 and 7 express all $b_{i, j, l}$ values as a function of $b_{i, 0,0}, p_{i, c}$ and $p_{i, e}$. With the following normalization condition imposed,

$$
\sum_{j=0}^{L_{\text {retry }}} \sum_{l=0}^{\mathrm{CW}_{j}-1} b_{i, j, l}=1 \text {, }
$$

finally $b_{i, 0,0}$ is given by (9):

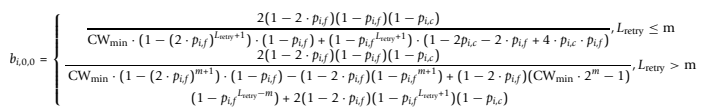

Since a given host transmits when its backoff timer reaches 0 , the probability that host $i$ transmits a packet in a randomly chosen slotted time, $\tau_{i}$, can be derived as:

$$
\tau_{i}=\sum_{j=0}^{L_{\mathrm{retry}}} b_{i, j, 0}=\sum_{j=0}^{L_{\mathrm{retry}}} p_{i, f}^{j} \cdot b_{i, 0,0}=b_{i, 0,0} \cdot \frac{1-p_{i, f}{ }^{L_{\mathrm{retry}}+1}}{1-p_{i, f}} .
$$

From Equation 10 we can see that $\tau_{i}$ depends on the probability of transmission failure $p_{i, f}$, which is determined with the collision probability $p_{i, c}$ and the corruption probability $p_{i}, e$. From Equations 2 to 4 and 9 and 10 , we can solve unknown parameters $\tau_{i}$ and $p_{i, f}$ numerically with a given set of frame size $\left(\mathrm{FS}_{1}, \mathrm{FS}_{2} \ldots\right.$
$\left.\mathrm{FS}_{K}\right)$ and BERs $\left(p_{1, b}, p_{2}, b \ldots p_{K, b}\right)$ corresponding to the $K$ hosts.

\subsection{Throughput analysis}

Let $P_{\operatorname{tr}}$ be the probability that at least one station transmits in the considered slotted time:

$$
P_{\mathrm{tr}}=1-\prod_{h=0}^{K-1}\left(1-\tau_{h}\right)
$$

Let $P_{i}$, single denote the probability that only host $i$ transmits and the remaining $K-1$ stations are idle on condition that at least one station transmits. Thus, it is expressed as:

$$
P_{i, \text { single }}=\tau_{i} \cdot \prod_{h=0, h \neq i}^{K-1}\left(1-\tau_{h}\right) /\left(1-\prod_{h=0}^{K-1}\left(1-\tau_{h}\right)\right) .
$$

Considering a given slot, the channel idle probability is $\left(1-P_{\mathrm{tr}}\right)$. The channel busy probability is $P_{\mathrm{tr}}$, which consists of the following parts: the probability of a successful transmission of host $i, P_{\mathrm{tr}} \cdot P_{i, \text { single }} \cdot p_{i, p s}$ the probability of a successful transmission of host $h(h \neq i)$, $P_{\mathrm{tr}} \cdot \sum_{h=0, h \neq i}^{K-1} P_{h, s}$ ingle $\cdot p_{h, p s} ;$ the probability of a failed transmission 
due to non-perfect channel conditions, $P_{\mathrm{tr}} \cdot \sum_{h=0}^{K-1} P_{h, s}$ ingle $\cdot\left(1-p_{h, p s}\right) ;$ and the probability $f$ a failed transmission due to collision, $P_{\mathrm{tr}} \cdot\left(1-\sum_{h=0}^{K-1} P_{h, \mathrm{si}}\right.$ ngle $)$. Hence the average length of a random slot normalized with the slotted time $T_{\text {slot }}, E[$ slot $]$ is derived from Equation13:

$$
\begin{aligned}
& E[\text { slot }]=\left(\left(1-P_{\mathrm{tr}}\right) \cdot T_{\mathrm{slot}}+P_{\mathrm{tr}} \cdot \sum_{h=0}^{K-1} P_{h, \mathrm{~s}} \text { ingle } \cdot\left(1-p_{h, e}\right) \cdot T s_{h}\right. \\
& \left.+P_{\mathrm{tr}} \cdot \sum_{h=0}^{K-1} P_{h, \text { single }} \cdot p_{h, e} \cdot T e_{h}+P_{\mathrm{tr}} \cdot\left(1-\sum_{h=0}^{K-1} P_{h, \text { si ngle }}\right) \cdot T_{\mathrm{C}}\right) / T_{\text {slot }}
\end{aligned}
$$

where $T s_{h}, T e_{h}$ are the time of host $h$ processing a successful transmission and experiencing a failed transmission due to a corruption respectively; $T_{\mathrm{C}}$ is the period of a collision. The values of $T s_{h}$ and $T c$ depend on the channel access mechanism. In case of the basic scheme, they can be expressed as:

$$
\begin{aligned}
& T s_{h}^{\mathrm{bas}}=\mathrm{DIFS}+H+T l_{h}+\gamma+\mathrm{SIFS}+\mathrm{ACK}+\gamma \\
& T c^{\mathrm{bas}}=\mathrm{DIFS}+H+T l^{*}+\gamma,
\end{aligned}
$$

and for the four-way handshaking scheme, they are:

$$
\begin{aligned}
& T s_{h}^{\mathrm{RTS}}=\mathrm{DIFS}+\mathrm{RTS}+\gamma+\mathrm{SIFS}+\mathrm{CTS}+\gamma+\mathrm{SIFS}+H+\mathrm{Tl}_{h}+\gamma+\mathrm{SIFS}+\mathrm{ACK}+\gamma \\
& T c^{\mathrm{RTS}}=\mathrm{DIFS}+\mathrm{RTS}+\gamma+\mathrm{SIFS}+\mathrm{CTS}+\gamma .
\end{aligned}
$$

$T e_{h}$ is equal to $T s_{h}$ in both of the basic and four-way handshaking scheme. DIFS, SIFS, $\mathrm{H}, \mathrm{ACK}$ and $\gamma$ denote DIFS time, SIFS time, the time to transmit the header, the time to transmit an ACK, and the time of propagation delay, respectively. $T l *$ is the time of the longest payload transmitted in a collision; $T l_{h}$ denotes the time of host $h$ transmitting its payload. It can be expressed as:

$$
T l_{h}=\mathrm{PL}_{h} \cdot 8 / r_{h}
$$

where $\mathrm{PL}_{h}$ is the payload length of host $h$ in bytes and $r_{h}$ is the used data rate of host $h$ for transmitting data packets.

The normalized saturation throughput of host $i, n S_{i}$, which is defined as the fraction of time that the channel is used for host $i$ to successfully transmit payload, can be expressed as:

$$
n S_{i}=\frac{P_{\mathrm{tr}} \cdot P_{i, \text { si ngle }} \cdot p_{i, p s} \cdot T l_{i}}{E[\text { slot }] \cdot T_{\text {slot }}} .
$$

Finally, the saturated throughput of host $i, S_{i}$ is thus given by:

$$
S_{i}=n S_{i} \cdot r_{i}
$$

\subsection{Delay analysis}

A delay for a successfully transmitted packet is defined as the duration from the time the packet is at the front of the MAC queue ready to be transmitted, until an acknowledgement informing this packet is received [12]. To calculate the average delay, the knowledge of packetdropping probability and average packet-dropping time is necessary. Let $P_{i \text {, drop }}$ denote the packet-dropping probability of host $i$. Since a packet is dropped if it encounters $L_{\text {retry }}+1$ failures, the probability $P_{i \text {, drop }}$ is equal to:

$$
p_{i, \text { drop }}=p_{i, f}{ }^{L_{\text {retry }}+1} .
$$

To host $i$, let $E_{i}\left[T_{\text {drop }}\right]$ be the average number of slots required for a packet to experience $L_{\text {retry }}+1$ failures in the $\left(0,1, \ldots, L_{\text {retry }}\right)$ stages. The average number of slots required for a packet waiting for transmission in the $j$ stage is $\left(\mathrm{CW}_{j}+1\right) / 2$, and thus $E_{i}\left[T_{\text {drop }}\right]$ can be expressed as:

$$
\begin{aligned}
& E_{i}\left[T_{\text {drop }}\right]=\sum_{j=0}^{L_{\text {rety }}} \frac{\mathrm{CW}_{j}+1}{2}= \\
& \left\{\begin{array}{c}
\frac{\mathrm{CW}_{\min } \cdot\left(2^{L_{\text {rety }}+1}-1\right)+L_{\text {retry }}+1}{2}, L_{\text {retry }} \leq m \\
\frac{\mathrm{CW}_{\text {min }} \cdot\left(2^{m+1}-1\right)+\mathrm{CW}_{\min } \cdot 2^{m} \cdot\left(L_{\text {retry }}-m\right)+\left(L_{\text {retry }}+1\right)}{2}, L_{\text {retry }}>m
\end{array}\right.
\end{aligned}
$$

The average number of slots required for host $i$ to successfully transmit a packet, $E_{i}[\mathrm{X}]$, is given by:

$$
E_{i}[X]=\sum_{j=0}^{L_{\text {retry }}}\left[\left(p_{i, f^{j}}-p_{i, \text { drop }}\right) \cdot \frac{\mathrm{CW}_{j}+1}{2}\right],
$$

where $\left(p_{i, f^{j}}-p_{i, \text { drop }}\right)$ is the probability that a packet that is not dropped reaches stage $j$. After calculation, Equation 19 becomes (20):

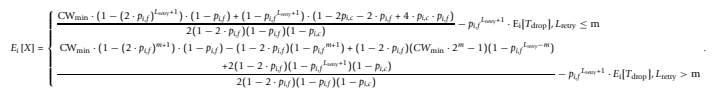

Thus the average packet delay of host $i, E_{i}[D]$, provided that this packet is not discarded, is then derived with Equations 13 and 20:

$$
E_{i}[D]=E_{i}[X] \cdot E[\text { slot }] .
$$

\section{Validations}

In this section, we conduct simulations to validate our analytical model. We adopt in this model the same 
parameters as those in the analytical model shown in Table 1. The IEEE 802.11 simulation model is developed using the $\mathrm{C}++$ programming language based on IEEE 802.11b standard (e.g., the initial backoff window is 32) [1]. For demonstration purposes, the data rate is 1 Mbps and the transmitting packet length is fixed as 1023 bytes. The packet is sent once every $10 \mathrm{~ms}$ to simulate a saturated traffic condition. Each result comes from the simulation of 100000 transmissions of packets.

In particular, we examine both the cases of equal and unequal channel conditions for transmitting stations. Figure 3 presents the simulation results and numerical results of the average throughputs of a station (the number of stations ranges from two to twenty) while all the transmitting stations are in perfect channel conditions (i.e., BER =0). As shown in the figure, we have a rather good match between the simulation results and analytical results (the maximum error is $1.89 \%$ ). Figure 4 shows the throughput of two stations individually in case that one station (denoted as IC host) is in perfect channel conditions while the other one (denoted as EC host) is in error-prone channels with the BER ranging from 0 to $8 \mathrm{E}-5$. It is observed in Figure 4 that when the channel conditions become more diverse, the difference between the simulation results and our analytical results generally are enlarged (the maximum error is $8.35 \%$ ). We think that the increasing error might be caused by the implementation of a statistical channel-error model using the event-driven simulation program (e.g., to implement BER on the occurrence of transmission failures). As illustrated in these figures, the analytical results and simulation results essentially can match pretty well. The simulation results demonstrate the accuracy of our analytical model.

\section{The numerical results and discussion}

In this section, we provide numerical results to demonstrate the unfairness of 802.11 DCF due to diverse channel conditions. The transmission scenario is as follows. Consider an 802.11b WLAN environment with two stations. Both the two stations transmit a saturated traffic flow with a fixed packet size using the basic CSMA/CA scheme. The adopted system parameters are presented in Table 1 . We provide performance analyses in both cases of stations transmitting at an equal data rate and at different data rates with a link adaptation mechanism. Then we use the Jain fairness index [3] associated with the analytical results to evaluate the fairness performance of IEEE 802.11 DCF. This index is represented as:

$$
\text { Jain fairness index }=\frac{\left(\sum_{i=1}^{K} x_{i}\right)^{2}}{K \sum_{i=1}^{K} x_{i}{ }^{2}}
$$

where $K$ is the number of stations. $x_{i}$ can be the throughput or delay associated with station $i$. The index

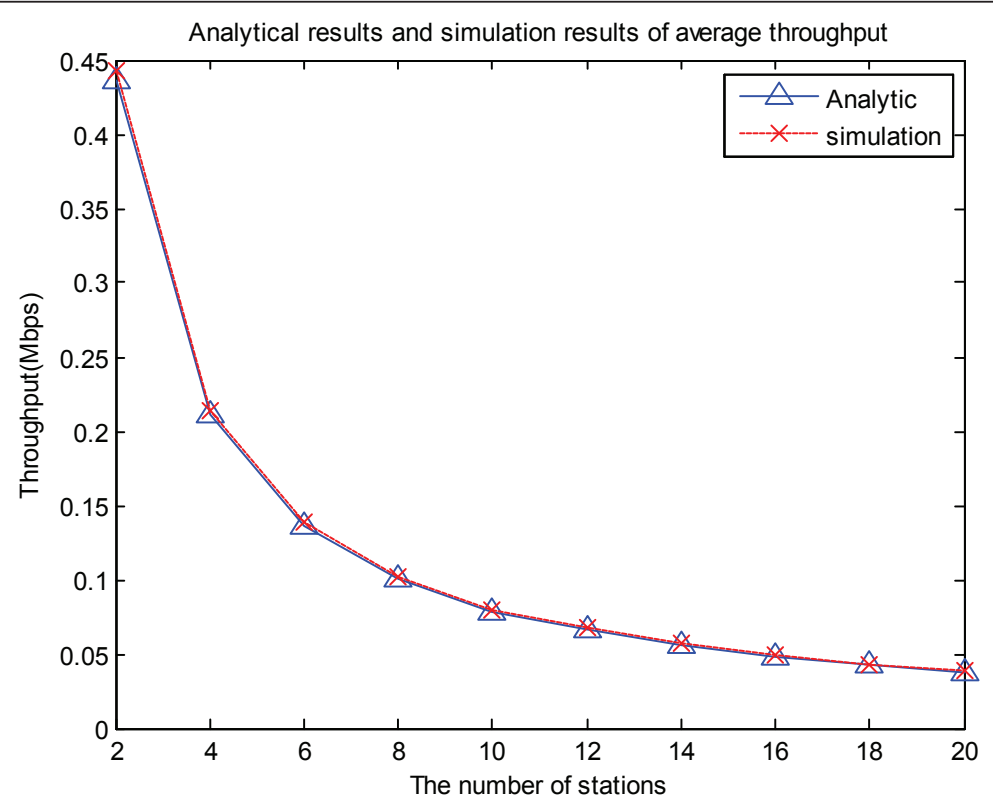

Figure 3 The comparison of simulation results vs. analytical results of throughput of an individual station varying with the number of stations (as the channels are in perfect conditions). 


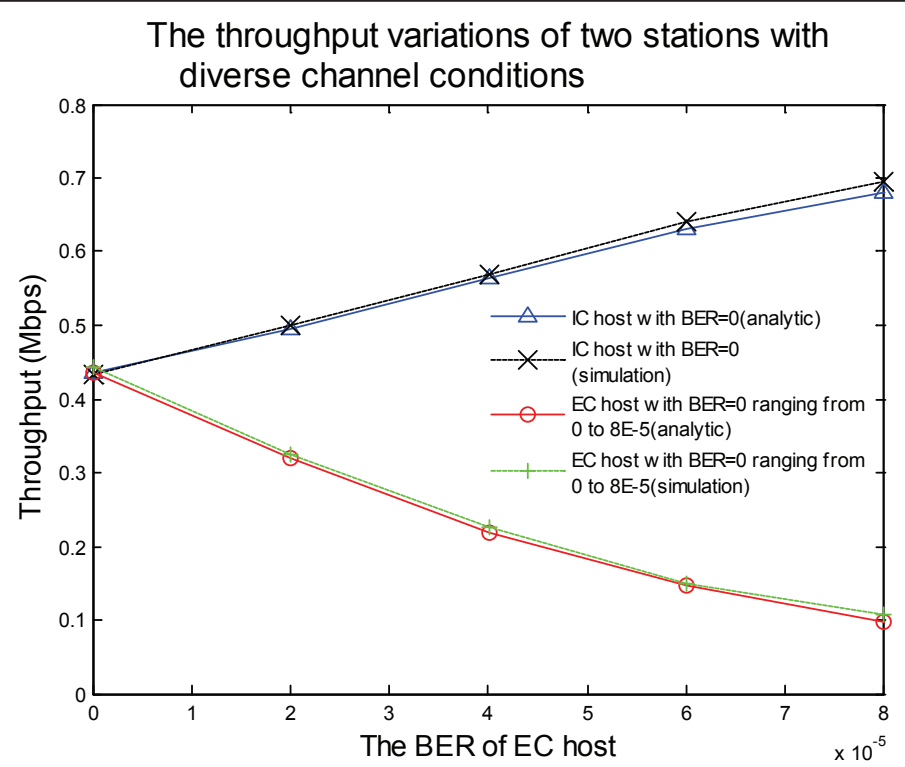

Figure 4 The comparison of simulation results vs. analytical results of throughput of two stations individually in case that one station (denoted as IC host) is in perfect channel conditions while the other one (denoted as EC host) is in error-prone channels with the BER ranging from 0 to $8 \mathrm{E}-5$

has a range of $(0,1]$ to evaluate fairness. Furthermore, we provide an example in Subsection 5.2 to show that applying the link adaptation cannot completely get rid of the effect of diverse link qualities due to limited MCSs available, and this can pose severe unfairness as we show in Subsection 5.1 and 5.3 later.

\subsection{Diverse link qualities with equal data rates}

First we analyze the scenario the hosts transmit at an equal data rate to demonstrate the unfairness due to diverse link qualities. Consider the two hosts use the same data rate of $1 \mathrm{Mbps}$. Assume one of them, named ideal-channel (IC) host, is always in a stationary and ideal channel condition (i.e., BER $=0$ ), whereas the other one, named error-prone-channel (EC) host, is initially in an ideal condition and later suffer from channel degradation due to the mobility with an average $\mathrm{BER}$ ranging from 0 to $8 \mathrm{E}-5$.

The saturated throughput and packet delay of each host are derived from Equations 16 and 21, respectively, and presented in Figures 5 and 6 with respect to the BER of EC host. It is shown that when the two hosts are in an ideal condition initially, their performances are equal no matter in terms of throughput or delay. When the BER of EC host deteriorates later, the performance variation of the two hosts is gradually enlarged. For instance if both the two hosts are in an ideal channel, the achievable throughput of each one is about 436 kbps as shown in Figure 5. In case EC host's BER deteriorates as $2 \mathrm{E}-5$, its throughput degrades to $319 \mathrm{kbps}$, whereas the throughput of IC host with ideal conditions increases to $494 \mathrm{Kbps}$. The performance variation is as large as $40.3 \%(176 \mathrm{kbps} / 436 \mathrm{kbps}=40.3 \%)$. The corresponding Jain fairness indices associated with throughput and packet delay are derived from Equation 22 and shown in Figures 7 and 8, respectively. It is also indicated that with the increasing difference of link qualities, fairness degrades as the indices associated with throughput and delay decrease from 1 to about 0.64 and 0.68 , respectively.

The performance variation arises by the following facts. Due to its higher BER, EC host averagely experiences more retries to succeed a transmission than IC host does. When a retransmission is performed, according to CSMA/CA standards, the backoff window size will be increased exponentially until the retries come to a certain limit. Thus EC host would averagely adopt a larger backoff timer and then has less chance to access the channel. Such the unfair behavior is similar to the scenarios of asymmetric information among nodes [39]. Our analytical results also demonstrated that when all the hosts transmit at an equal data rate, 802.11 CSMA/ CA can only present fairness on condition of homogeneous link qualities; the presence of diverse link qualities can cause significant unfairness.

\subsection{The discussion of diverse transmission qualities}

In this subsection, we provide an example to show that in the presence of diverse channel conditions, applying multiple data rates with a link adaptation mechanism 


\section{The average throughput of two stations in diverse channel conditions (with equal data rates)}

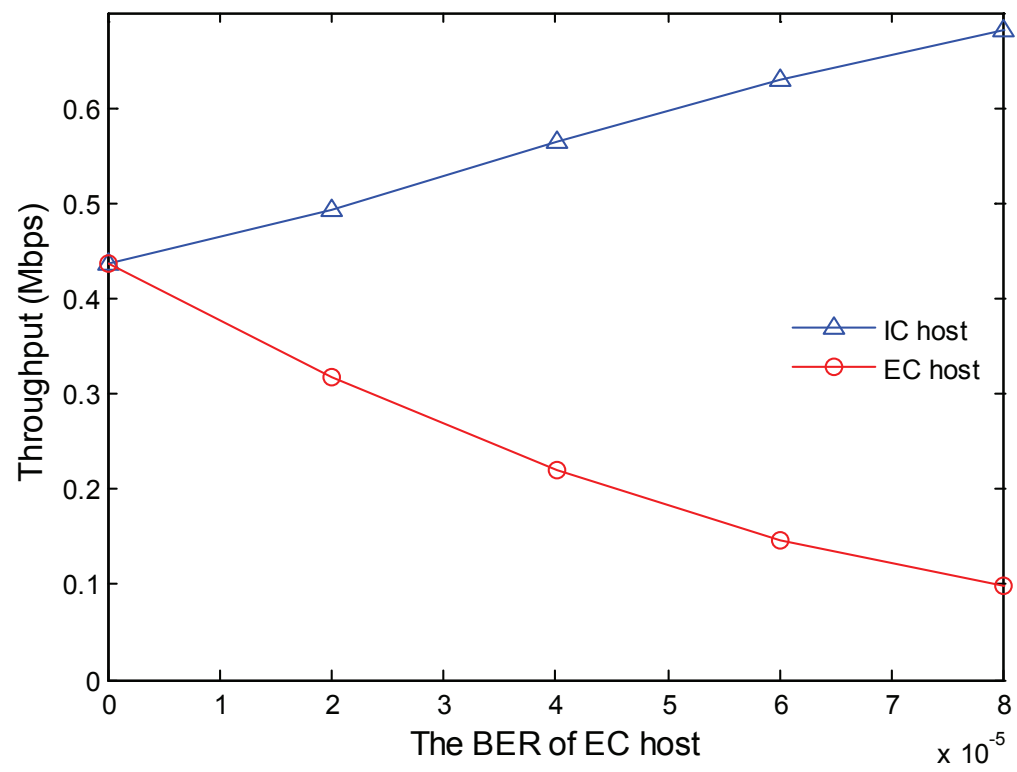

Figure 5 Throughput of IC host and EC host, respectively, when IC host is in perfect channel conditions while EC host is in errorprone channels with the BER ranging from 0 to $8 \mathrm{E}-5$ (the two nodes are with an equal data rate of $1 \mathrm{Mbps}$ ).

cannot completely equalize transmission qualities, thereby causing a similar unfair behavior as shown in Subsection 5.1. Consider IEEE 802.11b WLAN environments in which the MCSs available are uncoded differential binary phase shift keying (DBPSK), differential quadrature phase shift keying (DQPSK), complementary code keying 5.5 (CCK 5.5), and CCK 11 providing the data rate at $1,2,5.5$, and $11 \mathrm{Mbps}$, respectively. With a given SNR, the BER performed with these MCSs can be obtained empirically with experiments or

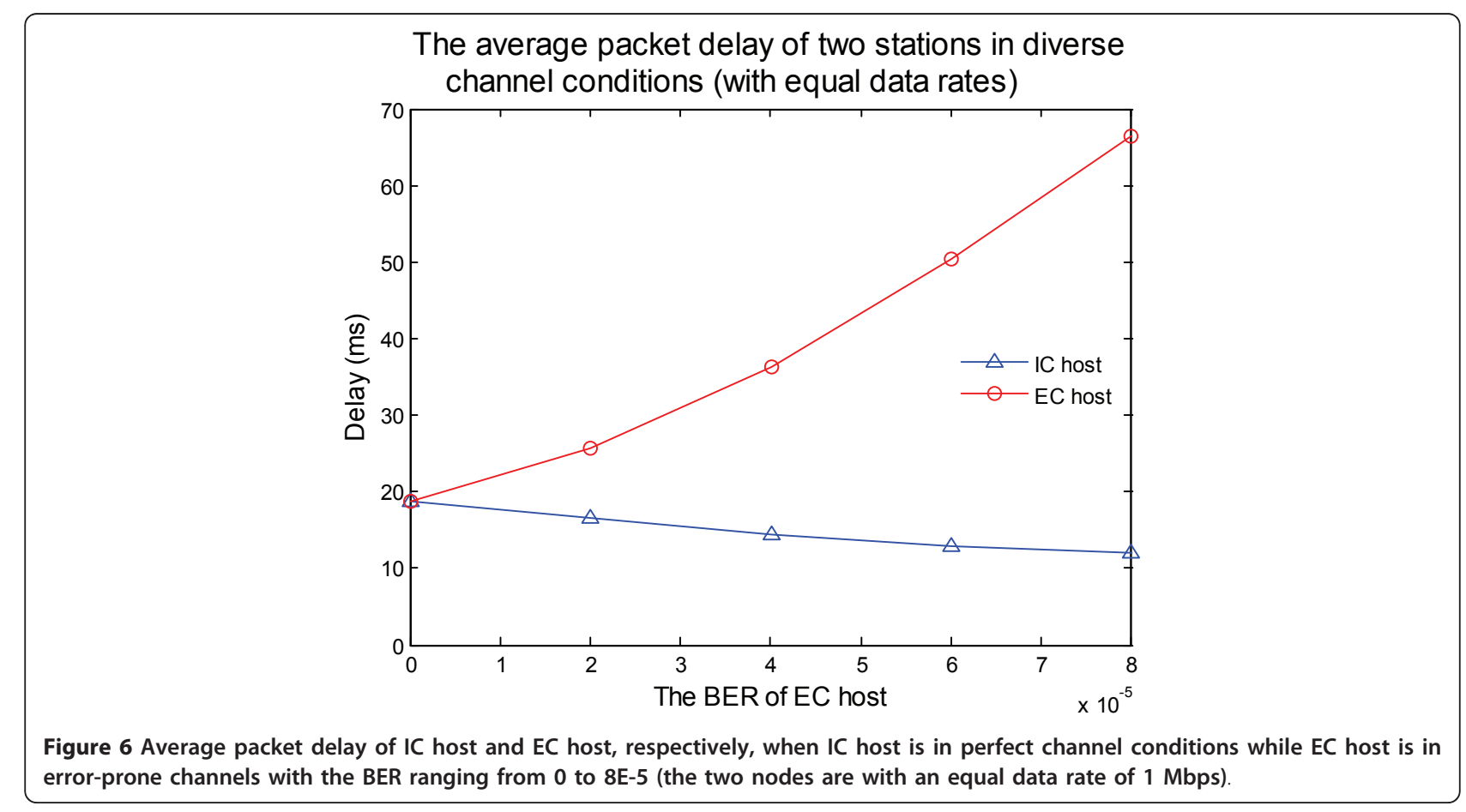




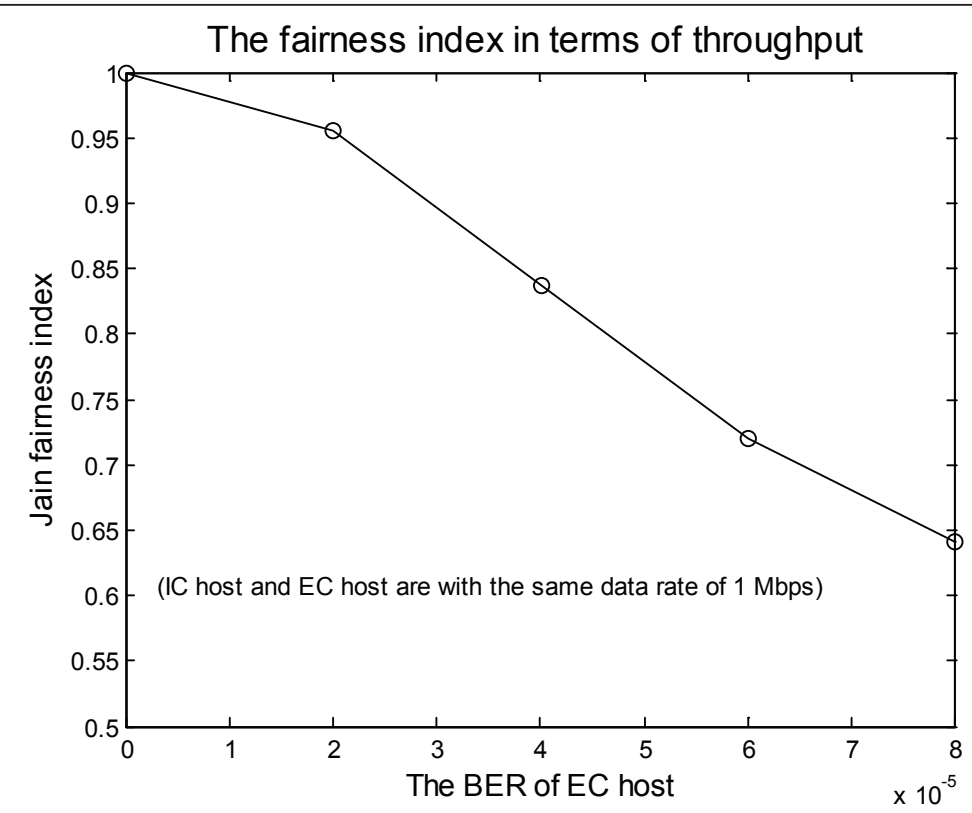

Figure 7 The Jain fairness index in terms of throughput with respect to the BER level of EC host (IC host and EC host are with an equal data rate of $1 \mathrm{Mbps}$ ).

theoretically with analyses. Figure 9 shows the BER vs. SNR of the $4802.11 \mathrm{~b}$ PHY modes provided empirically with Intersil WLAN product called HFA3861B in the environment with additive white Gaussian noise (AWGN) [40]. A link adaptation mechanism will dynamically select one MCS such that BER of the selected
MCS with the highest data rate is within a prescribed performance bound, e.g., a range less than $10^{-4}$ to $10^{-6}$ [41]. Consider that host A and host B have respectively experienced SNRs of 4 and $13 \mathrm{~dB}$ and both use the MCS of CCK 11 in a given time. Thus BERs for host $\mathrm{A}$ and host $\mathrm{B}$ are about $4 \times 10^{-2}$ and $1 \times 10^{-6}$,

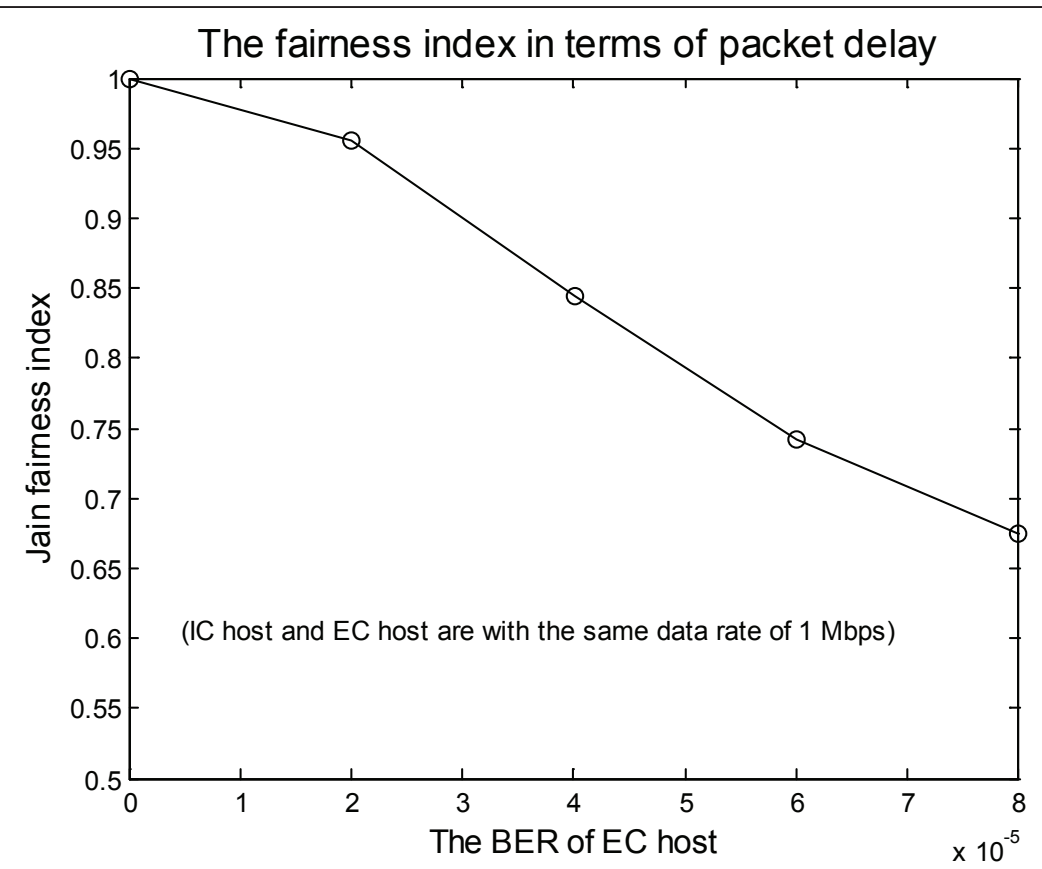

Figure 8 The Jain fairness index in terms of packet delay with respect to the BER level of EC host (IC host and EC host are with an equal data rate of $1 \mathrm{Mbps}$ ). 


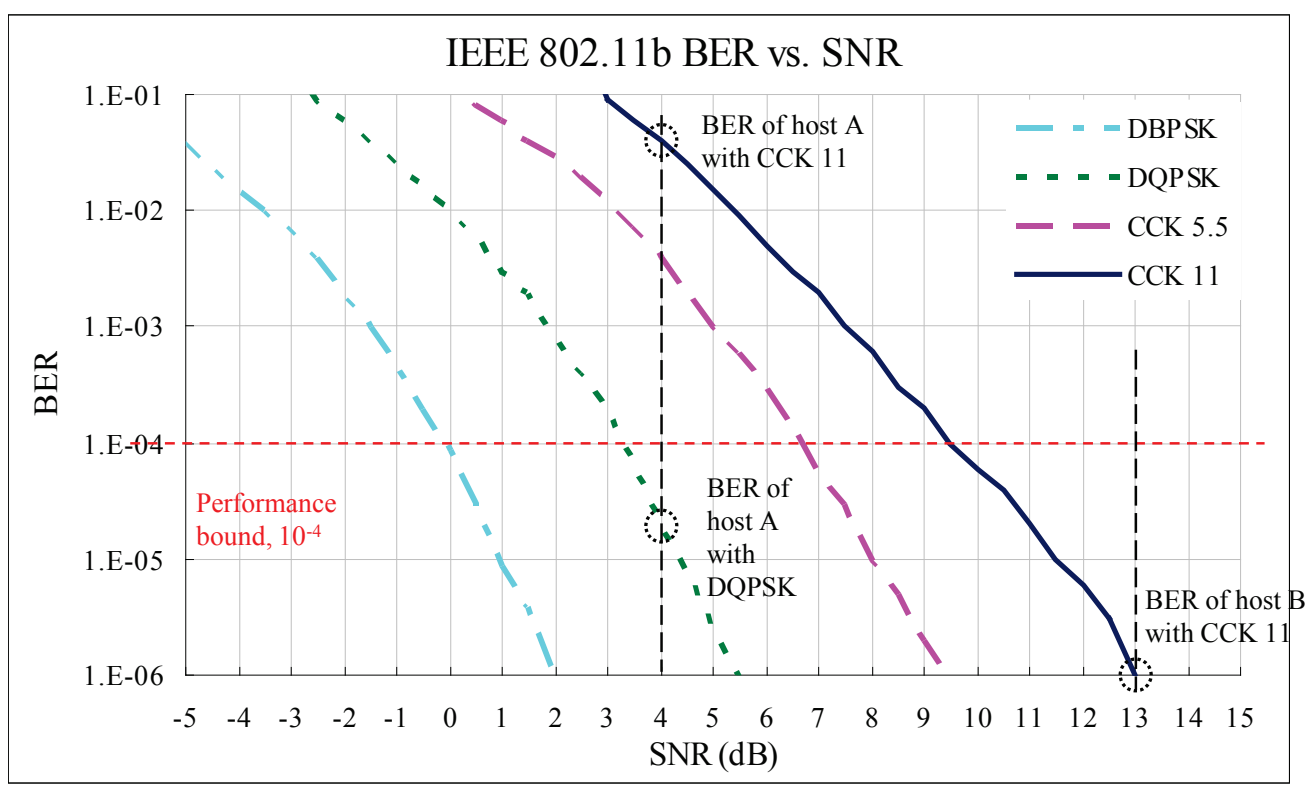

Figure 9 The BER performed with the 4 IEEE $802.11 \mathrm{~b}$ uncoded modulation schemes.

respectively, as shown in Figure 9, presenting a variation as large as about $4 \times 10^{4}$ times. By using the link adaptation scheme described above, MCS for host A changes to DQPSK with a much lower BER of $2 \times 10^{-}$ ${ }^{5}$. The link quality is effectively improved and the diversity of BER between hosts is greatly narrowed. However, the link qualities still present a certain difference of around 20 times, which can pose significant unfairness of channel sharing as we show in Subsection 5.3 later. From this example, it is shown that applying a link adaptation mechanism cannot completely get rid of the effect of diverse link qualities at most of the time due to limited MCSs available.

\subsection{Diverse link qualities with unequal data rates}

Now we use the scenario which hosts transmit at unequal data rates with a link adaptation mechanism for demonstrating the unfairness due to diverse link qualities. Consider that the link adaptation mechanism is applied with a performance bound, BER $<10^{-4}$. Assume that IC host transmits at a data rate of 1 Mbps in a stationary and perfect channel condition, whereas EC host transmits at $11 \mathrm{Mbps}$ in an ideal condition initially and later suffers from channel degradation with an average BER ranging from 0 to $8 \times 10^{-5}$. Figure 10 presents the saturated throughput of the two hosts. It is shown that when both of them are with ideal channel conditions initially, they present identical performances. This phenomenon is so called 'performance anomaly' [10] meaning that if at least one host transmits at a lower data rate, the throughput of the others at higher rates will be degraded below the level of the lower rate. The analytical results demonstrate that 802.11 CSMA/CA can present fairness regardless of the same or different data rates under the condition of homogeneous link qualities.

However, when the difference of channel conditions of the two hosts later enlarges gradually, the throughput of EC host suffers from more and more starvation whereas that of IC host remaining in a good condition is progressively increased. For example, if both the two hosts are in ideal conditions, their throughputs are equal as about $782 \mathrm{kbps}$. When the BER degrades to $4 \mathrm{E}-5$ later, EC host's throughput is extremely degraded to 320 Mbps whereas that of IC host is increased to $824 \mathrm{kbps}$. The throughput variation between the two stations is as large as $65 \%(504 / 782 \mathrm{kbps}=64.75 \%)$. Note that the throughput performance of a host does not correspond with its used data rates (i.e., the throughput of EC host using the data rate of $11 \mathrm{Mbps}$ is even lower than that of IC host with $1 \mathrm{Mbps}$ ) due to diverse link qualities. The corresponding Jain fairness index shown in Figure 11 also indicates that the throughput-based fairness gradually fades away such that the index decreases from 1 to about 0.64 . From these results, we show that the skewed performance of throughput and packet delay is caused by diverse link qualities rather than unequal data rates. The diverse link qualities can cause the severe unfairness to hosts either at an equal rate or at different rates with a link adaptation mechanism. 


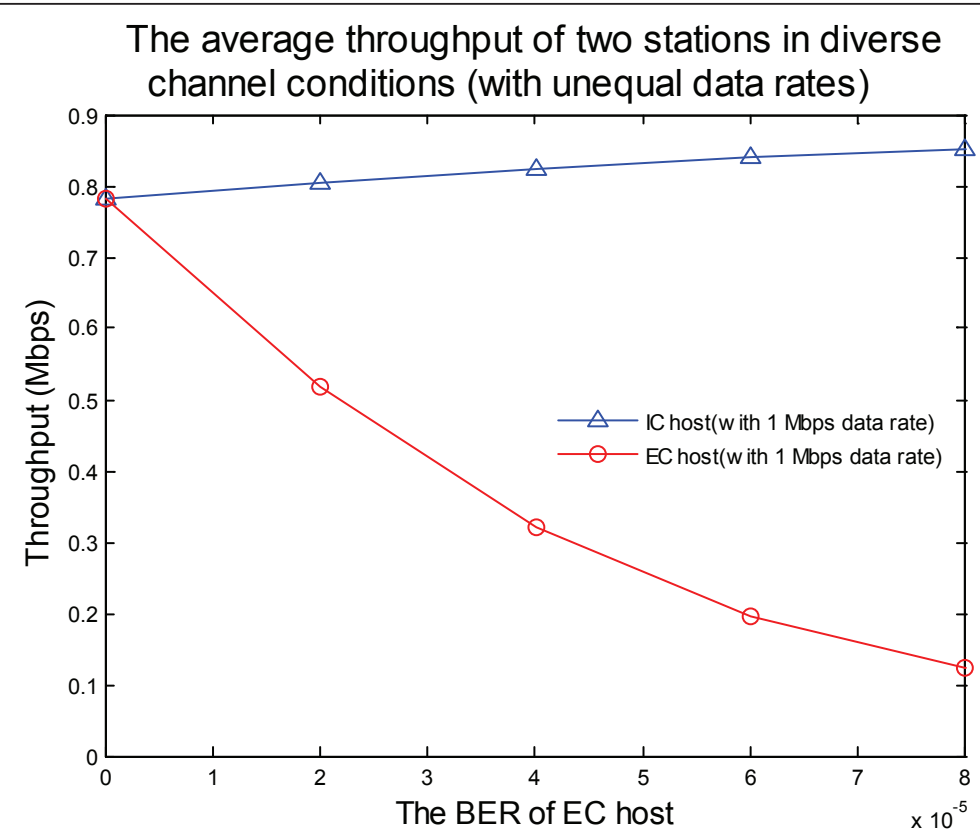

Figure 10 Throughput of IC host (with the data rate of $1 \mathrm{Mbps}$ ) and EC host (with $11 \mathrm{Mbps}$ ), respectively, when IC host is in perfect channel conditions while EC host is in error-prone channels with the BER ranging from 0 to $8 \mathrm{E}-5$.

\section{Conclusion}

In this article, we study the fairness of throughput and packet delays in IEEE 802.11 WLAN environments with diverse channel conditions. In this article, we exploit an analytical approach which extends a well-used two dimensional Markov chain model of DCF. From our analytical results, it is shown that 802.11 CSMA/CA can present fairness only provided that the link qualities of all the hosts are equal in a statistical average sense. It is also shown that the presence of diverse channel conditions can cause severe unfairness of channel sharing even with a link adaptation mechanism. We validate our

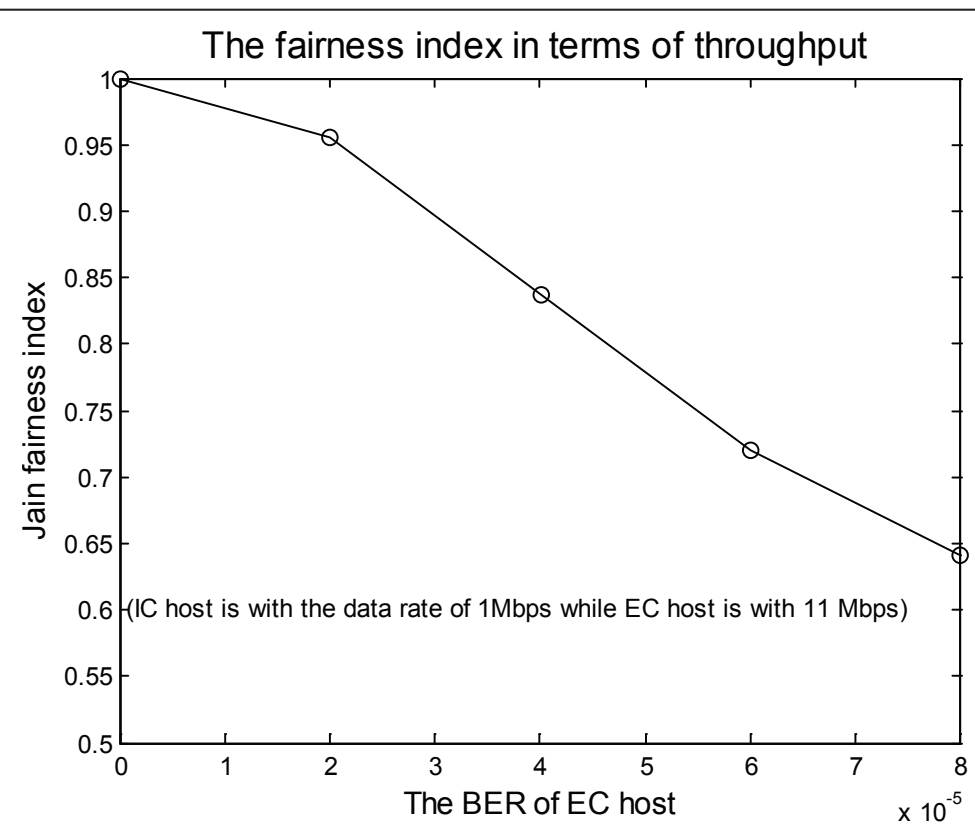

Figure 11 The Jain fairness index in terms of throughput with respect to the BER level of EC host (IC host is with the data rate of 1 Mbps while EC host is with $11 \mathrm{Mbps}$ ). 
analytical model via simulations and the results demonstrate its accuracy.

\begin{abstract}
Abbreviations
ACK: acknowledge; AIFS: arbitration inter-frame space; AP: access point: AWGN: additive white Gaussian noise; BDCF: bidirectional DCF; BER: bit error rate; CCK 5.5: complementary code keying 5.5; CSMA/CA: carrier sense multiple access with collision avoidance; CTS: clear-to-send; CW: contention windows; DBPSK: differential binary phase shift keying; DCC: distributed contention control; DCF: distributed coordination function; DIFS: DCF interframe space; DQPSK: differential quadrature phase shift keying; EC: errorprone-channel; EDCF: enhanced distributed coordination function; IC: idealchannel; MAC: medium access control; MCSs: modulation and coding schemes; PCF: point coordination function; PIFS: PCF inter-frame space; RTS: request-to-send; SIFS: short inter-frame space; SNR: signal-to-noise ratio; TCP: transmission-control-protocol; TXOP: transmission opportunity; UDP: userdatagram-protocol; WLAN: wireless local area networks.
\end{abstract}

\section{Acknowledgements}

This work was supported in part by Taiwan National Science Council under Grant 98-2221-E-003-009 and 99-2221-E-003-005.

\section{Author details}

'Department of Applied Electronic Technology, National Taiwan Normal University, Taipei, Taiwan ${ }^{2}$ Department of Electrical Engineering, Yuan Ze University, Taoyuan, Taiwan

\section{Competing interests}

The authors declare that they have no competing interests.

Received: 3 September 2010 Accepted: 19 July 2011

Published: 19 July 2011

\section{References}

1. IEEE $802.11 \mathrm{a} / \mathrm{b}$, Wireless LAN medium access control (MAC) and physical layer (PHY) specifications, standard IEEE, (August 1999)

2. NSP Nandiraju, H Gossain, D Cavalcanti, KR Chowdhury, DP Agrawa, Achieving fairness in wireless LANs by enhanced IEEE 802.11 DCF Proceedings of IEEE Conference on Wireless and Mobile Computing, Networking and Communications (WiMob), Montreal, Canada, 132-139, (June 2006)

3. CE Koksal, H Kassab, H Balakrishnan, An analysis of short-term fairness in wireless media access protocols, in Proceedings of ACM SIGMETRICS, California, USA, 118-119, (June 2000)

4. G Berger-Sabbatel, A Duda, O Gaudoin, M Heusse, F Rousseau, Fairness and its impact on delay in 802.11 networks, in Proceediings of IEEE GLOBECOM, Texas, USA, 2967-2973, (Nov-Dec 2004)

5. G Bianchi, Performance analysis of the IEEE 802.11 distributed coordination function IEEE J Sel Areas Commun. 18(3), 535-547 (2000). doi:10.1109/ 49.840210

6. E Ziouva, T Antonakopoulos, CSMA/CA performance under high traffic conditions: throughput and delay analysis Comput Commun. 25, 313-321 (2002). doi:10.1016/50140-3664(01)00369-3

7. Y Xiao, An analysis for differentiated services in IEEE 802.11 and IEEE 802.11 e wireless LANs, in Proceedings of IEEE International Conference on Distributed Computing Systems, Tokyo, Japan, 32-39, (March 2004)

8. IEEE 802.11e/D5.0, Draft Supplement to Part 11: wireless medium access control (MAC) and physical layer (PHY) specifications: medium access control (MAC) enhancements for quality of service (QoS), (June 2003)

9. S Choi, K Park, C Kim, Performance impact of interlayer dependence in infrastructure WLANs IEEE Trans Mobile Comput. 5(7), 829-845 (2006)

10. M Heusse, F Rousseau, G Berger-Sabbatel, A Duda, Performance anomaly of 802.11b, in Proceedings of IEEE INFOCOM, San Francisco, USA, 836-843, (March-April 2003)

11. Y Yang, M Marina, R Bagrodia, Experimental evaluation of application performance with 802.11 PHY rate adapatation mechanisms in diverse environments, in Proceedings of IEEE WCNC, Las Vegas, 2273-2278, (April 2006)
12. P Chatzimisios, AC Boucouvalas, $V$ Vitsas, IEEE 802.11 packet delay-a finite retry limit analysis, in Proceedings of IEEE GLOBECOM, San Francisco, USA, 950-954, (Dec. 2003)

13. SW Kim, BS Kim, Y Fang, Downlink and Uplink Resource Allocation in IEEE 802.11 Wireless LANs IEEE Trans Vehicul Technol. 54(1), 320-327 (2005). doi:10.1109/TVT.2004.838887

14. J Hu, K Erriksson, A new distributed contention control protocol for the IEEE 802.11 MAC layer, in Proceedings of the 1st International Conference on EBusiness and Telecommunication Networks, Setubal, Portugal, 393-401, (Aug. 2004)

15. BAHS Abeysekera, T Matsuda, T Takine, Dynamic contention window control to achieve fairness between uplink and downlink flows in IEEE 802.11 WLANs, in Proceedings of IEEE Wireless Communications and Networking Conference, Hong Kong, China, 2109-2114, (March 2007)

16. BAHS Abeysekera, T Matsuda, T Takine, Dynamic contention window control mechanism to achieve fairness between uplink and downlink flows in IEEE 802.11 wireless LANs IEEE Trans Mobile Comput. 7(9), 3517-3525 (2008)

17. M Heusse, F Rousseau, R Guillier, A Duda, Idle sense: an optimal access method for high throughput and fairness in rate changeful wireless LANS, in Proceedings of ACM SIGCOMM '05, vol. 35. Philadelphia, USA, 121-132

18. EL Aquilera, M Heusse, Y Grunenberger, F Rousseau, A Duda, J Casademont, An asymmetric access point for solving the unfairness problem in WLANs IEEE Trans Mobile Comput. 7(10), 1213-1227 (2008)

19. A Trunganont, $\vee V$ Visoottiviseth, Adaptive wireless bandwidth allocation for per-station fairness, in Proceedings of International Symposium on Communications and Information Technology (ISCIT), Incheon, Korea, 1286-1291, (Sept. 2009)

20. M Bredel, M Fidler, Understanding fairness and its impact on quality of service in IEEE 802.11, in Proceedings of IEEE INFOCOM, Rio de Janeiro, Brazil, 1098-1106, (April 2009)

21. N Khademi, M Othman, Guaranteeing per station and per flow fairness of downstream and upstream flows over IEEE 802.11 WLAN, in Proceedings of International Conference on Information and Multimedia Technology (ICIMT), Jeju Island, Korea, 431-435, (Dec. 2009)

22. G Berger-Sabbatel, A Duda, M Heusse, F Rousseau, Short-term fairness of 802.11 networks with several hosts, in Proceedings of Sixth IFIP IEEE International Conference on Mobile and Wireless Communication Networks, Paris, France, 263-274, (Oct. 2004)

23. CL Barrett, MV Marathe, DC Engelhart, A Sivasubramaniam, Analyzing the short-term fairness of IEEE 802.11 in wireless multi-hop radio networks, in Proceedings of IEEE International Conference on Modeling, Analysis, and Simulation of Computer and Telecommunications Systems, Texas, USA, 137-144, (Oct. 2002)

24. J He, HK Pung, A fairer multiple access protocol for multi-hop wireless networks: hybrid asynchronous time division multiple access protocol (HATDMA), in Proceedings of IEEE International Conference on Local Computer Network, Bonn/Königswinter, Germany, 356-365, (Oct. 2003)

25. Y Kwon, Y Fang, H Latchman, A novel MAC protocol with fast collision resolution for wireless LANS, in Proceedigns of IEEE INFOCOM, San Francisco, USA, 853-862, (March-April 2003)

26. T Nandagopal, TE Kim, X Gao, V Bharghavan, Achieving MAC layer fairness in wireless packet networks, in Proceedings of ACM International Symposium on Mobile Computing and Networking (MOBICOM), Boston, USA, 87-98, (Aug. 2000)

27. J Jeong, S Choi, CK Kim, Achieving Weighted Fairness between Uplink and Downlink in IEEE 802.11DCF-Based WLANs, in Proceedings of 2nd ACM International Conference on Quality of Service in Heterogeneous Wired/Wireless Networks (QShine), Lake Vista, Florida, USA, 10-22, (Aug. 2005)

28. M Bottigliengo, C Casetti, CF Chiasserini, M Meo, Smart traffic scheduling in 802.11 WLANs with access point, in Proceedings of IEEE VTC-Fall, Orlando, USA, 2227-2231, (Oct. 2003)

29. M Seyedzadegan, M Othman, S Subramaniam, Z Zukarnain, The TCP fairness in WLAN: a review, in Proceedings of IEEE Telecommunications and Malaysia International Conference on Communication, Penang, Malaysia, 644-648, (May 2007)

30. DJ Leith, P Clifford, D Malone, A Ng, TCP fairness in 802.11e WLANs. IEEE Commun Lett. 9(11), 964-966 (2005). doi:10.1109/LCOMM.2005.11004

31. EC Park, DY Kim, H Kim, CH Choi, A cross-layer approach for per-station fairness in TCP over WLANs IEEE Trans Mobile Comput. 7(7), 898-910 (2008) 
32. J Ha, CH Choi, TCP fairness for uplink and downlink flows in WLANs Proceedings of IEEE GLOBECOM, San Francisco, USA, (Nov.-Dec. 2006)

33. Y Wu, Z Niu, J Zheng, Study of the TCP upstream/downstream unfairness issue with per-flow queuing over infrastructure-mode WLANs Wireless Commun Mobile Comput. 5(4), 459-471 (2005). doi:10.1002/wcm.303

34. C Wang, T Lin, On fairness in heterogeneous WLAN environments, in Proceedings of IEEE GLOBECOM, San Francisco, USA, (Nov.-Dec. 2006)

35. Z Fang, B Bensaou, Y Wang, Performance evaluation of a fair backoff algorithm for IEEE 802.11 DFWMAC, in Proceedings of ACM International Symposium on Mobile Ad Hoc Networking \& Computing (MOBIHOC), Lausanne, Switzerland, 48-57, (June 2002)

36. S Deng, Capture effect in residential Ethernet LAN, in Proceedings of IEEE GLOBECOM, Singapore, 1678-1682, (Nov. 1995)

37. The Lucent Home Page. http://www.lucent.com/

38. D Qiao, S Choi, KG Shin, Goodput analysis and link adaptation for IEEE 802.11a wireless LANs IEEE Trans Mobile Comput. 1(4), 278-292 (2002). doi:10.1109/TMC.2002.1175541

39. V Kanodia, A Sabharwal, B Sadeghi, E Knightly, Ordered packet scheduling in wireless ad hoc networks: mechanisms and performance analysis Proceediings of ACM International Symposium on Mobile Ad Hoc Networking \& Computing (MOBIHOC), Lausanne, Switzerland, 58-70, (June 2002)

40. JdP Pavon, S Chio, Link adaptation strategy for IEEE 802.11 WLAN via received signal strength measurement, in Proceedings of IEEE ICC, Alaska, USA, 1108-1113, (May 2003)

41. Q Liu, S Zhou, GB Giannakis, Cross-Layer combining of adaptive Modulation and coding with truncated ARQ over wireless links IEEE Trans Wireless Commun. 3(5), 1746-1755 (2004). doi:10.1109/TWC.2004.833474

doi:10.1186/1687-1499-2011-42

Cite this article as: Wang et al.: Fairness analysis of throughput and delay in WLAN environments with channel diversities. EURASIP Journal on Wireless Communications and Networking 2011 2011:42.

\section{Submit your manuscript to a SpringerOpen ${ }^{\mathcal{O}}$ journal and benefit from:}

- Convenient online submission

- Rigorous peer review

- Immediate publication on acceptance

- Open access: articles freely available online

- High visibility within the field

- Retaining the copyright to your article

Submit your next manuscript at $\gg$ springeropen.com 